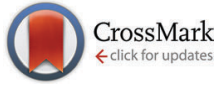

Cite this: Chem. Commun., 2014, 50, 10637

Received 12th May 2014

Accepted 2nd July 2014

DOI: $10.1039 / \mathrm{c} 4 \mathrm{cc} 03577 \mathrm{a}$

www.rsc.org/chemcomm

\section{Methoxy functionalisation: exerting synthetic control of the supramolecular and electronic structure of nitrogen-doped nanographenes $\dagger$}

\author{
Lankani P. Wijesinghe, $\ddagger^{\mathrm{ab}}$ Buddhie S. Lankage, $\ddagger^{\mathrm{ab}}$ Gearóid M. Ó Máille, ${ }^{\mathrm{ab}}$ \\ Sarath D. Perera, ${ }^{\mathrm{abc}}$ Deanne Nolan, ${ }^{\mathrm{ab}}$ Longsheng Wang ${ }^{\mathrm{ab}}$ and Sylvia M. Draper ${ }^{\star a b}$
}

We describe a series of functionalized $\mathrm{N}$-containing heterosuperbenzenes, created with a view to investigating the strategic role of methoxy substituents in (i) promoting cyclodehydrogenation and (ii) tuning the electronic properties and (iii) the supramolecular order in the resultant fused products.

The dominance of inorganic semiconductors in the manufacture of optoelectronic devices and field effect transistors is gradually being undermined by the emergence of functional organic materials. ${ }^{1-3}$ Although electron mobility remains a challenge, these represent lightweight, flexible alternatives that are more readily processed using solution-based methods. Within this arena, the opportunities proffered by the exceptional electronic and physical properties of graphene and its derivatives are undeniable. ${ }^{4-6}$

As many aspects in the formation of large graphitic sheets are difficult to control, synthetic chemists have pioneered routes to nanographenes with impressive and varying dimensions, and an array of peripheral substituents. ${ }^{7-10}$ Dependent on the latter are a range of material characteristics e.g. the nature of the $\pi-\pi$ interactions, HOMO-LUMO gaps and film-forming capabilities. ${ }^{11-14}$ The ability to manufacture graphene systems to such specificity is notably absent in exfoliation ${ }^{15} /$ epitaxial growth ${ }^{16}$ processes, and this makes the further development of bottom-up synthetic methodologies for the formation of next-generation and heteroatomcontaining graphenes all the more important. ${ }^{10,17}$

Oxidative cyclodehydrogenation is a key step in the chemical formation of planarised ring systems. All-carbon systems cyclise rapidly, while heteroatom-containing polyphenylenes appear to

\footnotetext{
${ }^{a}$ School of Chemistry, Trinity College, College Green, Dublin 2, Ireland. E-mail: smdraper@tcd.ie; Fax: +3531617 2826; Tel: +35318962026

${ }^{b}$ Centre for Research on Adaptive Nanostructures and Nanodevices (CRANN), Trinity College Dublin, Dublin 2, Ireland

${ }^{c}$ Chemistry Department, The Open University of Sri Lanka, Nugegoda, Sri Lanka $\dagger$ Electronic supplementary information (ESI) available: Experimental procedures and the characterization data for all new compounds, S1-S6. CCDC 976072 (2), 976383 (3) and 976036 (6). For ESI and crystallographic data in CIF or other electronic format see DOI: $10.1039 / \mathrm{c} 4 \mathrm{cc} 03577 \mathrm{a}$

\$ These authors contributed equally to the experimental data presented.
}

adopt a more complex mechanism for ring closure, e.g. both partially and fully fused species tend to be generated in a single reaction. ${ }^{18-20}$ Electron donating substituents both direct and promote CC bond formation under cyclodehydrogenation conditions. ${ }^{21,22}$

Tuning the intramolecular properties of graphenes is one step in the search for technology-enabling materials. Also essential is the ability to exert some supramolecular control of structure. Disc-like molecules tend to aggregate in columnar $\pi-\pi$ stacks which can be either enhanced or perturbed by peripheral units e.g. long chain alkyl substituted hexaperihexabenzocoronenes frequently exhibit liquid crystalline behaviour, ${ }^{2}$ whereas their iodo- or tert-butyl-functionalised derivatives give rise to Bernal-stacked crystalline dimers. ${ }^{23}$

This work reveals how the combination of heteroatom doping (as pioneered by Draper et al.), ${ }^{17,24-26}$ with H-bonding peripheral substituents, can unlock some exciting new applications for nanographene materials by controlling the outcome of the synthetic process and the HOMO-LUMO gaps and intermolecular order of the end-products.

The synthetic methods used to form the precursor polyphenylenes were modifications of published routes involving cyclopentadienones (generated via two-fold Knoevenagel condensations) and their subsequent [2+4] Diels Alder cycloadditions with either a di-pyrimidyl $(4 \mathrm{~N})^{17}$ or mono-pyrimidyl $(2 \mathrm{~N})$ acetylene (see ESI $\dagger$ ). These reactions resulted in the formation of the novel methoxy-substituted N-doped polyphenylenes $\mathbf{1}, \mathbf{3}$, and 5 which were subsequently subjected to oxidative cyclodehydrogenation. Via unpublished work, the authors have established that the analogous $2 \mathrm{~N}$ tert-butyl-containing systems (lacking methoxy substituents) would not fully cyclise under similar conditions.

The strategic positioning of peripheral methoxy groups is therefore key to generating this new series of nanographenes. The $\mathrm{FeCl}_{3}$-mediated oxidative cyclodehydrogenation of the 'para'methoxylated $\$ 1$ gave the new nitrogen heterosuperbenzene $\mathbf{2}$ (Scheme 1(a)).

Under the same conditions, reaction of 3 gave rise to the 'ortho'-methoxylated 4 (Scheme 1(b)), which proved challenging 
(a)

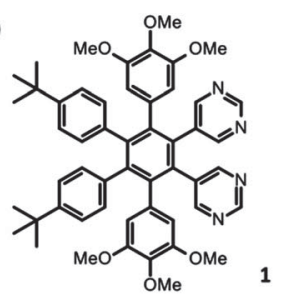

(i)

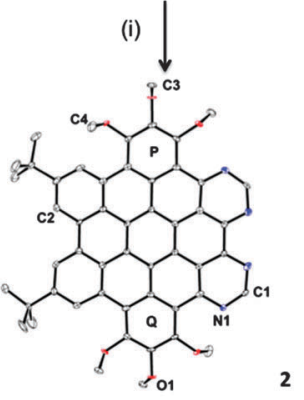

(b)
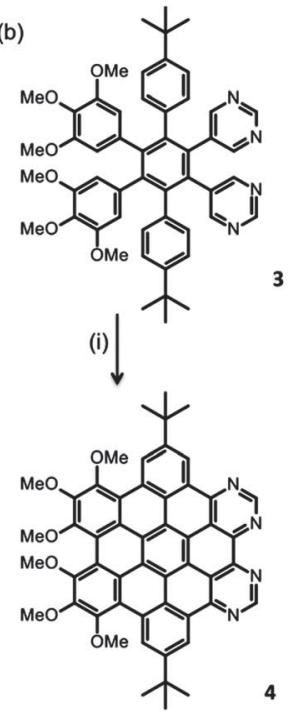

(c)
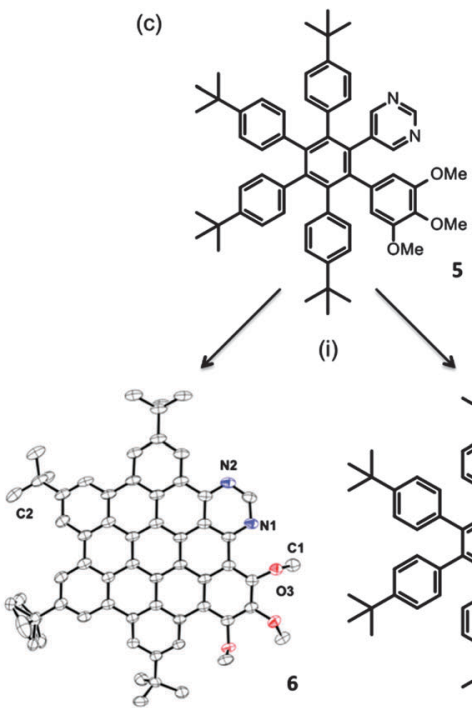

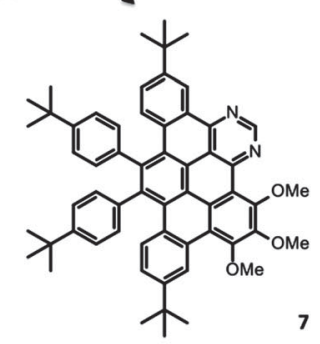

Scheme 1 The synthetic routes to $4 \mathrm{~N}$ (a and b) and $2 \mathrm{~N}$ (c) methoxy functionalized polyaromatic systems (i) $\mathrm{CH}_{2} \mathrm{Cl}_{2}, \mathrm{FeCl}_{3}\left(30\right.$ eq. in $\mathrm{CH}_{3} \mathrm{NO}_{2}$ ), $4 \mathrm{~h}$, (c) $24 \mathrm{~h}$; yields (a) 1: 88\%; 2: 53\% (b) 3: 82\%; 4: 35\% (c) 5: 54\%; 6: 40\%; 7: 10\%. Crystal structures are shown for 2 and 6 (hydrogens removed for clarity, thermal ellipsoids shown at $50 \%$ probability. $\mathbf{6}$ shows a disordered tert-butyl group). Atomic and ring labelling is to aid the crystallographic discussion (vide infra).

to purify due to the presence of a trace of the 5/6-cyclised product. Two products were isolated from the reaction of 5 under these conditions: the fully fused 6 ( $40 \%$ yield) and the co-synthesised half-cyclised 7 ( $10 \%$ yield). The products were all purified via column chromatography and on preparative plate thin layer chromatography. Full synthetic details and NMR analysis and discussion are available in the ESI. $\dagger$

2 and 6 crystallise in the monoclinic $P 2_{1} / c$ space group, with four molecules in the unit cell. Compound $\mathbf{4}$ unfortunately did not provide suitable crystals. Both show some distortion of the planar core due to steric congestion. In 2, for example, ring $\mathrm{P}$ is bent by $11.6^{\circ}$ with respect to the central phenyl ring (Scheme $1(\mathrm{a})$ ). In each of the rings $\mathrm{P}$ and $\mathrm{Q}$, two adjacent methoxy substituents point in the same direction (either above or below the central plane). The methoxy groups exist in a 'two up, one down' arrangement on each ring. This has a profound effect on the packing, resulting in offset head-to-tail dimers (AB) in a columnar stack (Fig. 1).

The $\pi \cdots \pi$ stacking is reflected in the short intradimer distance ( $\mathrm{A}-\mathrm{B}$ is $3.48 \AA$ ), while the interdimer distance is longer ( $A B-A B$ is $3.51 \AA$, see Fig. $1(a)$, right). The $A B$ dimers are not arranged in typical Bernal packing, but are offset by $1.25 \AA$. In addition to the $\pi \cdots \pi$ interactions, the dimers are held together by intermolecular hydrogen bonds between a methoxy-based methyl $\mathrm{H}$ and a methoxy $\mathrm{O}$ and a pyrimidyl $\mathrm{N}$ atom, $(\mathrm{O} 1 \cdots \mathrm{H}-\mathrm{C} 4$ $\left(3.53 \AA, 160.06^{\circ}\right)$ and $\left.\mathrm{N} 1 \cdots \mathrm{H}-\mathrm{C} 3\left(3.50 \AA, 149.43^{\circ}\right)\right)$.

The crystal structure of 6 is shown in Scheme 1(c) and in Fig. 1(b). The methoxy groups arrange themselves in a manner analogous to that observed in 2. Intramolecular H-bonding is observed (N1 ․ H-C1 (3.072 $\AA$ ) with a donor acceptor bond angle of $112.65^{\circ}$ ). 6 adopts a similar packing arrangement to 2, however the intradimer $\pi \cdots \pi$ distances are shorter $(3.45 \AA)$ with a larger offset $(3.12 \AA)$ and the distances between the dimers are slightly longer (3.64 ̊̊) (see Fig. 1(b)). In 2, the methoxy substituted rings of the dimeric molecules are above each other whereas in $\mathbf{6}$ they avoid each other. (a)

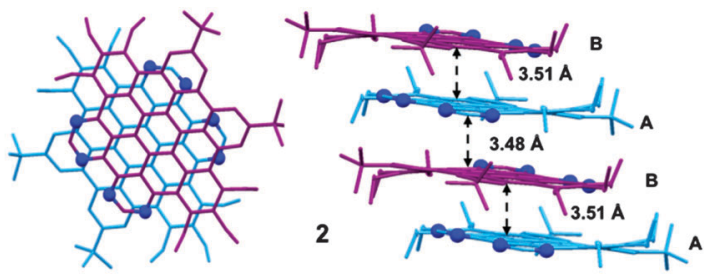

(b)
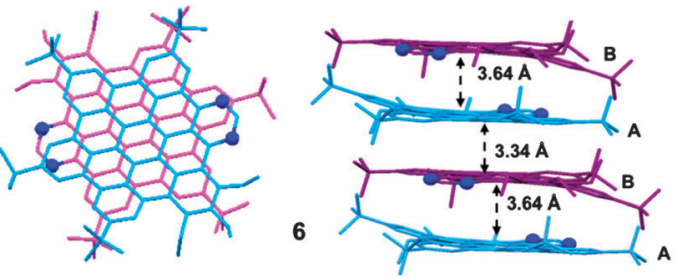

Fig. 1 X-Ray crystal structures of 2 and 6. (left) Head-to-tail packing arrangement and (right) side-view of the dimeric packing arrangement of (a) 2 and (b) 6

Fig. 2(a) compares the absorption spectra of compounds 2 , 4, 6 and 7 in toluene. Being the least aromatic compound in the series, 7 displays both the most hypsochromically shifted $\lambda_{\max }(305 \mathrm{~nm})$ and the broadest absorptions. The remaining members of the series display a prominent absorption at $\lambda_{\max }$ $355 \mathrm{~nm}$, as is typical of other benzenoids ${ }^{11}$ and fully fused N-HSB. ${ }^{17}$ The $\lambda_{\max }$ absorption in each case is relatively insensitive to both substitution and solvent polarity, and is characteristic of the $\beta$ band described by Clar. ${ }^{27}$ While $\beta$ bands are generally $\pi-\pi^{*}$ in character, a marked hypochromic shift in acidic medium points to some underlying $n-\pi^{*}$ contribution, as also seen in N-HSB. ${ }^{17}$ p-Bands, associated with electron relocalisation in the excited state, are also evident in the fullyfused systems $\mathbf{2}, \mathbf{4}$, and $\mathbf{6}$. They appear to be only moderately 
(a)
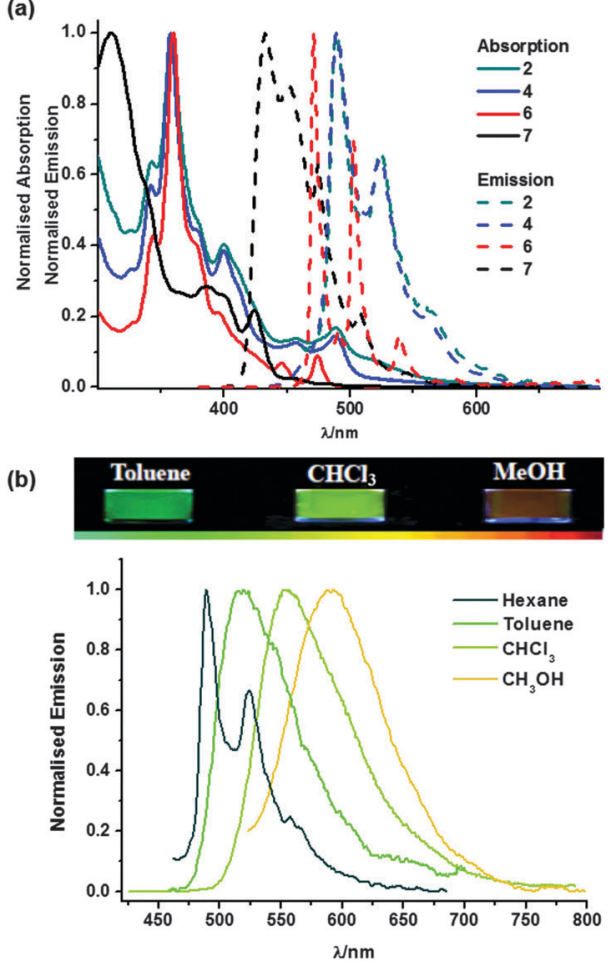

Fig. 2 (a) Normalised room temperature UV-Vis absorption (toluene) and emission (hexane) spectra of 2, 4, 6, and 7. (b) Normalised emission and photographic representation of the solvatochromic response of $\mathbf{4}$.

affected by substituent effects, being consistently centred around $\lambda \approx 400 \mathrm{~nm}$. This, coupled with a small hypochromic shift for this band in acidic medium (and the absorption's absence in the parent hydrocarbon) leads us to conclude that this band has significant $\mathrm{n}-\pi^{*}$ character. The lowest energy absorptions observed in each fully fused compound are the purely electronic, symmetry forbidden $\alpha(0-0)$ bands $(\lambda=470-500 \mathrm{~nm})$. The low symmetry (approximate $C_{2 \mathrm{v}}$ or less) of 2, 4 and 6 results in the enhanced intensity of these bands. ${ }^{28}$ From the transitions' energies we calculate HOMO-LUMO gaps of $2.48 \mathrm{eV}$ for $(4 \mathrm{~N}) 2$ and 4, and $2.60 \mathrm{eV}$ for $(2 \mathrm{~N}) 6$. This is reminiscent of the smaller band gap found in $\mathrm{N}$-containing systems of higher $\mathrm{N}$-doping by Bronner et al. ${ }^{29}$

The compounds are intensely fluorescent, and display prominent vibrational progressions in non-polar solvents. The most hypsochromically-shifted feature of the emission overlaps with the $\alpha$ absorption band, indicating minimal geometry change in the excited state. The partially unfused nature of 7 leads to the compound displaying the most blue-shifted emission of the series, at $\lambda_{\mathrm{em}} 432 \mathrm{~nm}$. While the molecule is not completely rigid, vibrational progressions of $c a .1200 \mathrm{~cm}^{-1}$ are observed in hexane. The compound also exhibits a short lifetime and the lowest emission quantum yield in the series (21\%, see Table S1 in ESI $\dagger$ ). Its fully fused counterpart (6) is red shifted due to its more aromatic nature $\left(\lambda_{\mathrm{em}}=470 \mathrm{~nm}, \tau=8.3 \mathrm{~ns}\right)$. Along with the other fully-fused compounds in the series, its rigidity results in prominent vibrational spacings of approximately $1350 \mathrm{~cm}^{-1}$. Fusion has also resulted in an increased emission quantum yield of $53 \%$ for the fully fused compound (6) and $32 \%$ for the $4 \mathrm{~N}$ compound (2).
Considering the donor-acceptor nature of these systems, it can be surmised that the initial locally-excited state can give rise to an intramolecular charge-transfer (ICT) state which is highly stabilised by polar solvents. This rationalises both the dramatic solvatochromic shifts observed, and the broadness of the emissions in methanol (Fig. 2(b) and Fig. S6 in ESI $\dagger$ ). Due to their higher inherent dipole moment, $\mathbf{2}$ and $\mathbf{4}$ give a far greater solvatochromic shift than $6\left(3500 \mathrm{~cm}^{-1}\right.$ and $3400 \mathrm{~cm}^{-1}$ respectively, compared with $\left.1578 \mathrm{~cm}^{-1}\right)$. The position of the MeO groups does not appear to affect the magnitude of the solvatochromic shift. Low temperature measurements were carried out on the compounds in $77 \mathrm{~K}$ butyronitrile glass. Each showed a moderate rigidochromic shift and a concomitant increase in emission lifetimes, as expected in rigid media (see Table S1 in ESI $\dagger$ ).

In order to gain further insight into the spectroscopic behaviour observed, cyclic voltammetry was employed to investigate the specific effects of -OMe functionalisation and N-doping on the systems' HOMO and LUMO levels. Each planarised compound shows a single irreversible oxidation process under positive potential. Compound 2 is the most readily oxidised, due to its six -OMe groups increasing the HOMO energy $\left(E_{\mathrm{p}}=0.65 \mathrm{~V} v s . \mathrm{Fc} / \mathrm{Fc}^{+}\right)$ (see Table $\mathrm{S} 1$ in $\mathrm{ESI} \dagger$ ). The lesser degree of -OMe functionalisation in 6 results in a more stabilised HOMO, giving a less facile oxidation $\left(E_{\mathrm{p}}=0.78 \mathrm{~V}\right)$. Although 7 also bears three-OMe groups, it presents the most difficult oxidation due to its lower aromaticity, which serves to further stabilise the HOMO $\left(E_{\mathrm{p}}=0.86 \mathrm{~V}\right)$. The reduction potentials are principally governed by the electronwithdrawing $\mathrm{N}$-atoms present: $6(2 \mathrm{~N})$ displays a reversible reduction wave at $E_{1 / 2}-2.02 \mathrm{~V}$, while $2(4 \mathrm{~N})$ is shifted to $E_{1 / 2}$ $-1.62 \mathrm{~V}$ due to its LUMO-stabilising four N-atoms. The hardest reduction is that of 7 , arising from its reduced aromaticity (which imparts a relatively destabilised LUMO). While low yields precluded the study of the electrochemical characteristics of $\mathbf{4}$, these data correspond well with that of the similar tert-butyl $\mathrm{N}$-HSB. ${ }^{18}$ In essence, these results show the fine-tuning of the available band gaps in these materials.

We have synthesised a novel set of methoxy-substituted $2 \mathrm{~N}$ and $4 \mathrm{~N}$-containing heterosuperbenzenes. CC bond formation and ring closure were promoted by incorporating strategically-placed and electron-donating methoxy substituents in the precursor polyphenylenes. Methoxy substitution has profoundly affected the supramolecular order observed in the crystalline nanographenes. The propensity for methoxy groups to engage in weak H-bonding appears to assist the molecules to orient in a head-to-tail arrangement within columnar $\pi-\pi$ stacks. The systems' HOMO-LUMO gap has been rendered smaller by the HOMO destabilising $\mathrm{MeO}$ groups and the LUMO stabilising $\mathrm{N}$-atoms. Importantly, this means that the electronic properties are under the control of the dopants and the peripheral units. In the search for small band gap materials this may negate the need to greatly extend the dimensions of the aromatic platform. Both band gap and inter-layer control are immensely important in investigations to broaden the application of nanographenes. This work points to the ability to control both parameters simply via chemical means.

We thank Drs J. O'Brien and M. Rüther for spectroscopic and technical assistance. We thank the Science Foundation Ireland 
for financial support (SFI 10/IN.I/12974, SFI 09/RFP/MTR2366). L.P.W. wishes to thank Trinity 1252 postgraduate award.

\section{Notes and references}

$\S$ The terms para and ortho are used here to describe the positioning of the 3,4,5-trimethoxybenzene units with regard to the central ring.

1 S. Allard, M. Forster, B. Souharce, H. Thiem and U. Scherf, Angew. Chem., Int. Ed., 2008, 47, 4070-4098.

2 S. Sergeyev, W. Pisula and Y. H. Geerts, Chem. Soc. Rev., 2007, 36, 1902-1929.

3 C. Li, M. Liu, N. G. Pschirer, M. Baumgarten and K. Müllen, Chem. Rev., 2010, 110, 6817-6855.

4 A. K. Geim and K. S. Novoselov, Nat. Mater., 2007, 6, 183-191.

5 M. J. Allen, V. C. Tung and R. B. Kaner, Chem. Rev., 2009, 110, 132-145.

6 K. S. Novoselov, V. I. Falko, L. Colombo, P. R. Gellert, M. G. Schwab and K. Kim, Nature, 2012, 490, 192-200.

7 D. J. Roberts, D. Nolan, G. M. Ö Máille, G. W. Watson, A. Singh, I. Ledoux-Rak and S. M. Draper, Dalton Trans., 2012, 41, 8850-8860.

8 M. Muller, C. Kubel and K. Müllen, Chem. - Eur. J., 1998, 4, 2099-2109.

9 L. Chen, Y. Hernandez, X. Feng and K. Müllen, Angew. Chem., Int. Ed., 2012, 51, 7640-7654.

10 M. Takase, T. Narita, W. Fujita, M. S. Asano, T. Nishinaga, H. Benten, K. Yoza and K. Mullen, J. Am. Chem. Soc., 2013, 135, 8031-8040.

11 R. Rieger and K. Müllen, J. Phys. Org. Chem., 2010, 23, 315-325.

12 R. Yamaguchi, S. Ito, B. S. Lee, S. Hiroto, D. Kim and H. Shinokubo, Chem. - Asian J., 2013, 8, 178-190.

13 D. J. Jones, B. Purushothaman, S. Ji, A. B. Holmes and W. W. H. Wong, Chem. Commun., 2012, 48, 8066-8068.
14 D. J. Gregg, C. M. A. Ollagnier, C. M. Fitchett and S. M. Draper, Chem. - Eur. J., 2006, 12, 3043-3052.

15 X. Li, X. Wang, L. Zhang, S. Lee and H. Dai, Science, 2008, 319, 1229-1232.

16 C. Berger, Z. Song, X. Li, X. Wu, N. Brown, C. Naud, D. Mayou, T. Li, J. Hass, A. N. Marchenkov, E. H. Conrad, P. N. First and H. W. A. de, Science, 2006, 312, 1191-1196.

17 S. M. Draper, D. J. Gregg and R. Madathil, J. Am. Chem. Soc., 2002, 124, 3486-3487.

18 D. J. Gregg, E. Bothe, P. Hoefer, P. Passaniti and S. M. Draper, Inorg. Chem., 2005, 44, 5654-5660.

19 A. Graczyk, F. A. Murphy, D. Nolan, V. Fernandez-Moreira, N. J. Lundin, C. M. Fitchett and S. M. Draper, Dalton Trans., 2012, 41, 7746-7754.

20 P. Rempala, J. Kroulik and B. T. King, J. Org. Chem., 2006, 71, 5067-5081.

21 B. T. King, J. Kroulik, C. R. Robertson, P. Rempala, C. L. Hilton, J. D. Korinek and L. M. Gortari, J. Org. Chem., 2007, 72, 2279-2288.

22 Z. Wang, F. Doetz, V. Enkelmann and K. Müllen, Angew. Chem., Int. Ed., 2005, 44, 1247-1250.

23 P. T. Herwig, V. Enkelmann, O. Schmelz and K. Müllen, Chem. - Eur. J., 2000, 6, 1834-1839.

24 S. M. Draper, D. J. Gregg, E. R. Schofield, W. R. Browne, M. Duati, J. G. Vos and P. Passaniti, J. Am. Chem. Soc., 2004, 126, 8694-8701.

25 C. J. Martin, B. Gil, S. D. Perera and S. M. Draper, Chem. Commun., 2011, 47, 3616-3618.

26 A. Graczyk, F. A. Murphy, D. Nolan, V. Fernandez-Moreira, N. J. Lundin, C. M. Fitchett and S. M. Draper, Dalton Trans., 2012, 41, 7746-7754.

27 E. Clar, Polycyclic Hydrocarbons, Academic Press, 1964, vol. 2.

28 W. Hendel, Z. H. Khan and W. Schmidt, Tetrahedron, 1986, 42, 1127-1134.

29 C. Bronner, S. Stremlau, M. Gille, F. Brauße, A. Haase, S. Hecht and P. Tegeder, Angew. Chem., Int. Ed., 2013, 52, 4422-4425. 